\title{
Correction to: Molecular detection and clinicopathological characteristics of advanced/recurrent biliary tract carcinomas harboring the $F G F R 2$ rearrangements: a prospective observational study (PRELUDE Study)
}

Yuta Maruki $^{1}$ - Chigusa Morizane $^{1}\left(\mathbb{D} \cdot\right.$ Yasuhito Arai $^{2} \cdot$ Masafumi Ikeda $^{3} \cdot$ Makoto Ueno $^{4} \cdot$ Tatsuya Ioka $^{5}$. Atsushi Naganuma ${ }^{6} \cdot$ Masayuki Furukawa $^{7}$. Nobumasa Mizuno ${ }^{8} \cdot$ Tadashi Uwagawa $^{9}$ Naminatsu Takahara ${ }^{10}$. Masashi Kanai $^{11}$ - Akinori Asagi $^{12}$ - Satoshi Shimizu ${ }^{13} \cdot$ Atsushi Miyamoto $^{14}$ - Seigo Yukisawa ${ }^{15}$.

Makoto Kadokura $^{16}$ - Yasushi Kojima ${ }^{17}$. Junji Furuse ${ }^{18}$ - Takako Eguchi Nakajima ${ }^{19,20} \cdot$ Kentaro Sudo $^{21}$. Noritoshi Kobayashi $^{22}$ - Natsuko Hama ${ }^{2}$. Takeharu Yamanaka ${ }^{23}$ - Tatsuhiro Shibata ${ }^{2}$ Takuji Okusaka ${ }^{1}$

Published online: 30 December 2020

(C) Japanese Society of Gastroenterology 2020

\section{Correction to: J Gastroenterol} https://doi.org/10.1007/s00535-020-01735-2

In the original publication of the article, the supplementary figure 2 was published wrongly. The correct

The original article can be found online at https://doi.org/10.1007/ s00535-020-01735-2.

\section{Chigusa Morizane}

cmorizan@ncc.go.jp

1 Department of Hepatobiliary and Pancreatic Oncology, National Cancer Center Hospital, 5-1-1 Tsukiji, Chuo-ku, Tokyo 104-0045, Japan

2 Division of Cancer Genomics, National Cancer Center Research Institute, Tokyo, Japan

3 Department of Hepatobiliary and Pancreatic Oncology, National Cancer Center Hospital East, Kashiwa, Japan

4 Department of Gastroenterology, Hepatobiliary and Pancreatic Medical Oncology Division, Kanagawa Cancer Center, Kanagawa, Japan

5 Department of Oncology Center, Yamaguchi University Hospital, Yamaguchi, Japan

6 Department of Gastroenterology, National Hospital Organization Takasaki General Medical Center, Gunma, Japan

7 Department of Hepato-Biliary-Pancreatology, National Hospital Organization Kyushu Cancer Center, Fukuoka, Japan

8 Department of Gastroenterology, Aichi Cancer Center Hospital, Aichi, Japan

9 Department of Surgery, The Jikei University School of Medicine, Tokyo, Japan

10 Department of Gastroenterology, Graduate School of Medicine, The University of Tokyo, Tokyo, Japan supplementary figure 2 has been updated in Springer Link.

The original article has been corrected.

11 Department of Therapeutic Oncology, Graduate School of Medicine, Kyoto University, Kyoto, Japan

12 Department of Gastrointestinal Medical Oncology, National Hospital Organization Shikoku Cancer Center, Ehime, Japan

13 Department of Gastroenterology, Saitama Cancer Center, Saitama, Japan

14 Department of Surgery, National Hospital Organization Osaka National Hospital, Osaka, Japan

15 Department of Medical Oncology, Tochigi Cancer Center, Tochigi, Japan

16 Department of Gastroenterology, Kofu Municipal Hospital, Yamanashi, Japan

17 Department of Gastroenterology, National Center for Global Health and Medicine, Tokyo, Japan

18 Department of Medical Oncology, Kyorin University Faculty of Medicine, Tokyo, Japan

19 Department of Clinical Oncology, St.Marianna University School of Medicine, Kanagawa, Japan

20 Kyoto Innovation Center for Next Generation Clinical Trials and iPS Cell Therapy, Kyoto University Hospital, Kyoto, Japan

21 Division of Gastroenterology, Chiba Cancer Center, Chiba, Japan

22 Department of Oncology Division, Yokohama City University School of Medicine, Kanagawa, Japan

23 Department of Biostatistics, Yokohama City University Graduate School of Medicine, Kanagawa, Japan 